\title{
GMR
}

\section{Isolation and characterization of polymorphic microsatellite markers in the endangered species Bretschneidera sinensis Hemsl.}

\author{
M. Li, H.F. Chen, Z.F. Wang and S. Zhang \\ Key Laboratory of Vegetation Restoration and Management of Degraded \\ Ecosystems \& Guangdong Provincial Key Laboratory of Applied Botany, \\ South China Botanical Garden, Chinese Academy of Sciences, Guangzhou, \\ China \\ Corresponding authors: H.F. Chen / Z.F. Wang \\ E-mail: h.f.chen@scbg.ac.cn / wzf@scib.ac.cn
}

Genet. Mol. Res. 15 (3): gmr.15038234

Received March 7, 2016

Accepted April 25, 2016

Published August 18, 2016

DOI http://dx.doi.org/10.4238/gmr.15038234

Copyright $(\odot 2016$ The Authors. This is an open-access article distributed under the terms of the Creative Commons Attribution ShareAlike (CC BY-SA) 4.0 License.

\begin{abstract}
Bretschneidera sinensis is an endangered species that is mainly distributed in South China. As a tertiary relict and the single species in the Bretschneideraceae family, it has a high conservation value. To investigate the influence of human disturbance on its mating system, 63 new microsatellites were developed using restriction-site-associated DNA sequencing and their polymorphisms were tested on 30 samples from one population. Among the 63 microsatellites, the number of alleles per locus ranged from 2 to 16 . The observed and expected heterozygosities ranged from 0.133 to 0.967 and from 0.127 to 0.912 , respectively. These microsatellites may be used for studying the mating system of $B$. sinensis as well as the within-population hereditary structure.
\end{abstract}

Key words: Bretschneideraceae; Conservation genetics; Genetic diversity; Genetic markers; Mating system; RAD-seq 


\section{INTRODUCTION}

Bretschneidera sinensis is a tree species mainly distributed in South-east China, extending to northern Thailand and northern Vietnam. It grows up to 10-20 $\mathrm{m}$ tall in low to middle elevation forest. As a tertiary relict and the single species in the family Bretschneideraceae, it is of high research value. Due to habitat loss and logging, $B$. sinensis is becoming increasingly rare. According to recent studies, most populations generally contain less than 30 individuals (Xu et al., 2013). In the field, this species also suffers from reproductive failure due to low seed productivity and seed germination rates (Qiao et al., 2011; Liang et al., 2013), which further increasing its extinction risk. At present, it is included in the China national key protected wild plants and the China plant red data book (Fu and Jin, 1992), and is listed as endangered by the international union for conservation of nature and natural resources (Sun, 1998).

$B$. sinensis has hermaphroditic flowers that form long racemes and are mainly pollinated by hymenopteran insects (Qiao et al., 2012). A pollination ecology study showed that $B$. sinensis has a mating system consisting of a mixture of outcrossing and selfing (Qiao et al., 2012). The mating system plays a key role in maintaining genetic diversity within populations. However, it is currently unknown how such mating systems are influenced by habitat fragmentation and the reduced population size in $B$. sinensis. In the present study, we report 63 newly developed polymorphic microsatellite loci for $B$. sinensis that may be used in future mating system studies.

\section{MATERIAL AND METHODS}

A total of 31 leaf tissues of $B$. sinensis were sampled from two populations: 30 from Nan-Kun Mountain Nature Reserve (NKMNR, 113 $48^{\prime} 41^{\prime \prime}-113^{\circ} 56^{\prime} 32^{\prime \prime} \mathrm{E}, 23^{\circ} 35^{\prime} 14^{\prime \prime}-23^{\circ} 43^{\prime} 05^{\prime \prime} \mathrm{N}$ ) in Guangdong Province and one from Hua-Ping National Nature Reserve (HPNNR, 109 48'54"-109 $58^{\prime 2} 20^{\prime \prime}$ E, $25^{\circ} 31^{\prime} 10^{\prime \prime}-25^{\circ} 39^{\prime} 36^{\prime \prime} \mathrm{N}$ ) in Guangxi Province. For microsatellite locus identification, the HPNNR sample and one sample randomly chosen from NKMNR were used to construct restriction-siteassociated DNA sequencing (RAD-seq) library. Total genomic DNA was exacted from these two tissues. The extraction was performed using a modified CTAB method (Doyle, 1991). The two DNA samples were then subjected to RAD-seq using a HiSeq2500 sequencer (Illumina Inc., San Diego, CA, USA). After trimming the adapters and removing low-quality sequences, we obtained 21,533,218 and 23,378,532 clean sequences from the two DNA samples, respectively. The sequences were then assembled using STACK v. 1.24 (Catchen et al., 2011, 2013). From the assembled sequences, a total of 417 microsatellites (di- and trinucleotides; containing at least seven repeats) were identified using MSATCOMMANDER v. 0.8.2 (Faircloth, 2008) of which 211 could be used for further primer design.

Polymerase chain reaction (PCR) amplifications were conducted for the 211 sequences for the NKMNR individual in a $20-\mu \mathrm{L}$ volume containing $2 \mu \mathrm{L} 10 \mathrm{X}$ PCR buffer $\left(\mathrm{Mg}^{2+}\right.$ plus), $0.5 \mathrm{mM}$ dNTPs, $0.4 \mu \mathrm{M}$ each primer, $50 \mathrm{ng}$ DNA template, and $1 \mathrm{U}$ Taq polymerase (TaKaRa, Biotechnology Co., Ltd., Daliang, China). The PCR amplification conditions were as follows: initial template denaturation at $95^{\circ} \mathrm{C}$ for $5 \mathrm{~min}$; then 35 cycles of denaturation at $94^{\circ} \mathrm{C}$ for $30 \mathrm{~s}$, annealing at $55^{\circ} \mathrm{C}$ for $30 \mathrm{~s}$, and elongation at $72^{\circ} \mathrm{C}$ for $45 \mathrm{~s}$; followed by a final extension at $72^{\circ} \mathrm{C}$ for $10 \mathrm{~min}$. The amplified products were electrophoresed on a $2 \%$ agarose gel. A total of 170 sequences successfully amplified the target regions.

Six NKMNR individuals were preliminary used to examine the polymorphism of the 170 microsatellite loci. The amplified PCR products were electrophoresed on an ABI 3730 sequencer (Applied Biosystems, Carlsbad, CA, USA), and their sizes were measured using the ABI GeneMapper software v. 4.1. The loci that showed clear and stable polymorphisms were further used for allelic variation assessments on 30 individuals from NKMNR (Table 1).

Genetics and Molecular Research 15 (3): gmr.15038234 
Table 1. Characteristics and genetic diversity of 63 microsatellite primers developed in Bretschneidera sinensis.

\begin{tabular}{|c|c|c|c|c|c|c|c|c|}
\hline Locus & Repeat motif & Primer sequences $\left(5^{\prime}-3^{\prime}\right)$ & Size range (bp) & 5'-fluorescence label & $N_{\mathrm{A}}$ & $H_{\mathrm{O}}$ & $H_{\mathrm{E}}$ & $F$ \\
\hline BLS-3 & $(\mathrm{TA})_{9}$ & F:AGGAAGAAATCAAACAGGATTTGG & $158-170$ & HEX & 5 & 0.633 & 0.672 & 0.0589 \\
\hline & & R:TGTTAGCACCTACTACCAAGGG & & & & & & \\
\hline BLS-5 & $(\mathrm{CT})_{12}$ & F:TGACTACTTTCTGCTTGCCAC & $220-256$ & FAM & 11 & 0.900 & 0.887 & -0.0149 \\
\hline & & R:ACTCGTTGCCTAATCTCTGAAAC & & & & & & \\
\hline BLS-6 & $(\mathrm{CT})_{12}$ & F:TACCCGATAAGCCCTGTGG & $144-166$ & FAM & 7 & 0.870 & 0.736 & -0.1860 \\
\hline & & R:TGGAAATCGCAGAGGAATTAGG & & & & & & \\
\hline BLS-9 & $(\mathrm{GA}) 9$ & F:CCATCCATTCCCTTTGAATTTCTG & $167-169$ & HEX & 2 & 0.276 & 0.242 & -0.1429 \\
\hline & & R:GAGCCGAGATGGGCAGATG & & & & & & \\
\hline BLS-10 & $(\mathrm{TA})_{9}$ & F:TCTGTAGTGGTTCAATATTAAGTGG & $179-183$ & FAM & 3 & 0.429 & 0.512 & 0.1660 \\
\hline & & R:TCTTGGGATATAGACCAGAAATACG & & & & & & \\
\hline BLS-12 & $(\mathrm{TC})_{9}$ & F:CTCGCACATTGGCCATTCC & $222-224$ & FAM & 2 & 0.448 & 0.506 & 0.1165 \\
\hline & & R:ACATGTGATGTGAGTACAACATGG & & & & & & \\
\hline BLS-13 & $(\mathrm{AC})_{11}$ & F:TGTCGATGTGAGTTTGCCTTA & $206-214$ & FAM & 3 & 0.600 & 0.518 & -0.1613 \\
\hline & & R:CATCTCAATACAATTCAAGCATCA & & & & & & \\
\hline BLS-15 & $(\mathrm{TC})_{12}$ & F:ATAATGCAGAATCCACACCACTT & $237-245$ & FAM & 5 & 0.733 & 0.727 & -0.0087 \\
\hline & & R:GAGGGGTTCAAAATGAGTAACAG & & & & & & \\
\hline BLS-52 & (AT)9 & F:CTCGAGCTTTGCTTGAAAGG & $225-243$ & HEX & 7 & 0.704 & 0.825 & 0.1497 \\
\hline 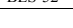 & (R11) & R:GATGCCGAACTTGGCTCTG & $225=-275$ & IL & & & & \\
\hline BLS-53 & $(\mathrm{AT})_{8}$ & F:GTATCCTAGTTTGGCTGGCG & $230-256$ & HEX & 12 & 0.821 & 0.753 & -0.0933 \\
\hline & & R:GCTTCCCTGTTCTTGTAAGATTCG & & & & & & \\
\hline BLS-57 & $(\mathrm{CT})_{13}$ & F:GCATGCATATTGGCTCTTTACG & $258-268$ & HEX & 5 & 0.667 & 0.663 & -0.0061 \\
\hline & & R:TGTCACATGCACAAGAATGG & & & & & & \\
\hline BLS-61 & $(\mathrm{CT})_{8}$ & F:TCGATTTGCTTCTAATGCACCC & $168-172$ & FAM & 3 & 0.636 & 0.532 & -0.2025 \\
\hline & & R:CCCTTCCCACCAGAGATTCC & & & & & & \\
\hline BLS-63 & $(\mathrm{CT})_{11}$ & F:ACACTTAACACCTCTACTTGGTTG & $208-220$ & HEX & 4 & 0.724 & 0.745 & 0.0289 \\
\hline & & R:TGGCATAACTCAAGTGTACAGG & & & & & & \\
\hline BLS-66 & (CT) 8 & F:TCTCTTGCCACGCGATTTC & $211-219$ & HEX & 4 & 0.633 & 0.606 & -0.0465 \\
\hline & & $\mathrm{R}: G G A C T G G C C A A G G A A G G G$ & & & & & & \\
\hline BLS-67 & $(\mathrm{CT})_{8}$ & F:GACTTGTACATGCCGAAGGTG & $258-266$ & HEX & 3 & 0.700 & 0.656 & -0.0684 \\
\hline & & R:CAGTGGAAATAGCCAACATTGC & & & & & & \\
\hline BLS-71 & $(\mathrm{AG})_{9}$ & F:TCTTAAGTTGCAGGCCGAC & $211-237$ & HEX & 12 & 0.967 & 0.869 & -0.1139 \\
\hline & & R:AGCAACATCTTCCTAGTGAGTTC & & & & & & \\
\hline BLS-73 & $(\mathrm{CT})_{10}$ & F:CCGCTGGGTCCTCCATAG & $188-196$ & FAM & 3 & 0.276 & 0.246 & -0.1228 \\
\hline & & R:TCAAGAATTTGAATCAGTAGCAACC & & & & & & \\
\hline BLS-74 & $(\mathrm{AG}) 10$ & F:GAAGCAAGAGTTCTCTGCCG & $190-192$ & FAM & 2 & 0.567 & 0.508 & -0.1179 \\
\hline & & R:GCTCTTAGTACCGAATCATGGC & & & & & & \\
\hline BLS-75 & $(\mathrm{CT}) 8$ & F:TCAAACTGCTGCAAAGAGGC & $169-173$ & FAM & 3 & 0.552 & 0.482 & -0.1487 \\
\hline & & R:TTAAAGCACCCGAAAGCCC & & & & & & \\
\hline BLS-76 & $(\mathrm{AT})_{12}$ & F:TGACCGGTATTTGCATAAAGG & $259-276$ & HEX & 9 & 0.500 & 0.793 & $0.3738^{*}$ \\
\hline & & R:CTCAACACACCCTTCCATTGAG & & & & & & \\
\hline BLS-85 & $(\mathrm{CT})_{8}$ & F:TCACAGGCACAATACCCTAATTTC & $177-183$ & FAM & 4 & 0.667 & 0.572 & -0.1682 \\
\hline & & R:GGCGGCTCCATTGACTTAC & & & & & & \\
\hline BLS-94 & $(\mathrm{AC})_{9}$ & F:ACAAGCAACAGGTTGTCAGG & $192-202$ & HEX & 5 & 0.552 & 0.738 & 0.2558 \\
\hline & & R:CAGGTTCAATTCCAGTCGGC & & & & & & \\
\hline BLS-98 & $(\mathrm{AG}) 9$ & F:GAGAGAGGAGTCTTGATCTTTGC & $264-308$ & HEX & 4 & 0.481 & 0.587 & 0.1826 \\
\hline & & R:GTCATGGAAGCAGGCGTTG & & & & & & \\
\hline BLS-100 & $(\mathrm{AC})_{9}$ & F:GCTACAAATGCCATCCGC & $261-279$ & HEX & 7 & 0.786 & 0.749 & -0.0504 \\
\hline & & R:TGGCAGCTTGACAGAATGG & & & & & & \\
\hline BLS-112 & (CT)s 8 & F:GGTCCACTCACAAGATGAGC & $255-257$ & HEX & 2 & 0.367 & 0.305 & -0.2083 \\
\hline & & R:TGTAGTTGTCGCTCCTTCC & & & & & & \\
\hline BLS-118 & $(\mathrm{AC})_{8}$ & F:CTTCTTCATTCTTCAGTTCCCAAG & $225-227$ & HEX & 2 & 0.133 & 0.127 & -0.0545 \\
\hline & & R:GCTCAAACATCTGTAGCCCAAG & & & & & & \\
\hline BLS-121 & $(\mathrm{AC}) 12$ & F:ACCTACTATAAACCCGTTCAGTC & $168-228$ & FAM & 16 & 0.690 & 0.912 & 0.2473 \\
\hline & & R:GTGTTCGAAATCAAATAGGATGGC & & & & & & \\
\hline BLS-122 & $(\mathrm{AT})_{7}$ & F:CTGAATGGGTGGTAGGTCATC & $168-172$ & FAM & 3 & 0.200 & 0.244 & 0.1812 \\
\hline & & R:GCAACCTGTCCTGTGAACC & & & & & & \\
\hline BLS-123 & (AT)8 & F:ACCATGCTTGATTATGCACCC & $178-180$ & FAM & 2 & 0.467 & 0.499 & 0.0667 \\
\hline & & R:GTTTGCACACCATGCCTTC & & & & & & \\
\hline BLS-128 & $(\mathrm{ATC}) 9$ & F:CGACATTTCGCTCTGTAAAGCAC & $129-156$ & FAM & 5 & 0.759 & 0.696 & -0.0922 \\
\hline & & R:CAGACCAGGATCTTGATTTCGG & & & & & & \\
\hline BLS-130 & $(\mathrm{AG})_{13}$ & F:TTATCTACTATAGGACTTCCAGCC & $211-265$ & HEX & 9 & 0.933 & 0.857 & -0.0907 \\
\hline & & R:TGTTCCGCTTATTGCTGCC & & & & & & \\
\hline BLS-133 & $(\mathrm{AG})_{8}$ & F:GCTTGTTTGGTGACCTGGG & $210-222$ & HEX & 3 & 0.200 & 0.188 & -0.0675 \\
\hline & & R:GGATGCACATCTTCCAAACTATTC & & & & & & \\
\hline BLS-134 & (CT) 9 & F:GGTCAAATCGACAAATAACGACTG & $218-226$ & HEX & 5 & 0.250 & 0.736 & $0.6643^{*}$ \\
\hline & & R.ATCCTAAAGCTCTAATACCATGAAG & & & & & & \\
\hline BLS-145 & $(\mathrm{AAG})_{6}$ & F:TTTCACCGTCCGATTTGTTC & $180-213$ & FAM & 3 & 0.633 & 0.538 & -0.1799 \\
\hline & & R:AGTTTGGGTGCACGGATTG & & & & & & \\
\hline BLS-149 & $(\mathrm{GAT})_{7}$ & F:AGTGTAATTCATCCTTCGACTTG & $161-176$ & FAM & 3 & 0.333 & 0.412 & 0.1933 \\
\hline & & R:CTCCCATCTAATTTCCCACCTTC & & & & & & \\
\hline BLS-152 & $(\mathrm{CT})_{10}$ & F:AGGGAGGTTCAATCAATTCCTG & $196-198$ & FAM & 2 & 0.517 & 0.509 & -0.0169 \\
\hline & & R:AGCAGCAGACTTATGCATTCC & & & & & & \\
\hline BLS-153 & $(\mathrm{CT})_{9}$ & F.GAAACCACCTTGGCCCTTG & $167-171$ & FAM & 3 & 0.467 & 0.429 & -0.0899 \\
\hline & & R:CTCTCTCAAATCGCGGGTATC & & & & & & \\
\hline BLS-154 & $(\mathrm{AT})_{8}$ & F:TCCCATCAGTTCATGTTGTACG & $258-260$ & HEX & 2 & 0.286 & 0.444 & 0.3609 \\
\hline & & R:ACTTAAGCCCGATTTATGCAAG & & & & & & \\
\hline BLS-156 & $(\mathrm{AT}) 10$ & F:GCGGCTGAAACCTATGCAG & $301-337$ & HEX & 6 & 0.759 & 0.742 & -0.0224 \\
\hline & & R:AGAGATTTGTTTGGACCGCC & & & & & & \\
\hline BLS-170 & $(\mathrm{AG})_{12}$ & F:TGAAGCATTAATTTGATACGCCC & $235-253$ & HEX & 9 & 0.733 & 0.819 & 0.1058 \\
\hline & & $\mathrm{R}: \mathrm{GACCAGTGCCATCAATCCC}$ & & & & & & \\
\hline BLS-172 & $(\mathrm{AG})_{12}$ & F·AGCTACAAGATGGTTGGGC & $227-233$ & HEX & 3 & 0267 & 0377 & 02970 \\
\hline & & R:CACTCGTAACAACTGCACCC & & & & & & \\
\hline BLS-174 & $(\mathrm{CT})_{11}$ & F.ATCTTGGTCACCTCCTCAAG & $166-170$ & FAM & 3 & 0.483 & 0.623 & 02276 \\
\hline & & R:CAACTCATCGGCTCGTTGC & & & & & & \\
\hline BLS-175 & $(\mathrm{ATC})_{6}$ & F:TCCCTGATGAACGGTCACG & $177-186$ & FAM & 2 & 0.333 & 0.364 & 0.0852 \\
\hline & & R:CCTTTCGCCGGCAATCTAC & & & & & & \\
\hline BLS-181 & $(\mathrm{CT})_{7}$ & F:TTACTGTGCAGCCGATCCC & $180-186$ & FAM & 3 & 0.300 & 0.422 & 0.2927 \\
\hline & & R:GCCAGAGCCAATATGGTTGC & & & & & & \\
\hline BLS-183 & (AAG)8 8 & F:CACCAGTTTCCAAGACCGC & $256-271$ & HEX & 6 & 0.793 & 0.755 & -0.0514 \\
\hline
\end{tabular}




\begin{tabular}{|c|c|c|c|c|c|c|c|c|c|}
\hline Locus & Repeat motif & Primer sequences $\left(5^{\prime}-3^{\prime}\right)$ & Size range (bp) & 5'-fluorescence label & $N_{\mathrm{A}}$ & $H_{\mathrm{O}}$ & $H_{\mathrm{E}}$ & $F$ & EMBL accession No. \\
\hline & & R:AGGCGGGTCTTCAGTTGAG & & & & & & & \\
\hline \multirow[t]{2}{*}{ BLS-186 } & $(\mathrm{CT})_{13}$ & F:CTCAGCTCAAGCCTTTGCC & $141-157$ & FAM & 8 & 0.889 & 0.829 & -0.0740 & LN886597 \\
\hline & & R:CACAACAAGTGTTACTGCTTTGG & & & & & & & \\
\hline \multirow[t]{2}{*}{ BLS-193 } & $(\mathrm{AC})_{9}$ & F:ACCAAACCGCAATGGCTAC & $240-296$ & HEX & 8 & 0.593 & 0.787 & $0.2505^{*}$ & LN886598 \\
\hline & & R:TCAAGTGGGTTCGAGTACTTTG & & & & & & & \\
\hline \multirow[t]{2}{*}{ BLS-195 } & $(\mathrm{AT})_{13}$ & F:AACAGAGGGCAGTTGTCAG & $203-257$ & HEX & 14 & 0.933 & 0.884 & -0.0573 & LN886599 \\
\hline & & R:AAACAGGCCTCCCACAAAG & & & & & & & \\
\hline \multirow[t]{2}{*}{ BLS-202 } & $(\mathrm{AG})_{14}$ & F:GGGTTTGGTTAACTGGTGGC & $215-229$ & HEX & 5 & 0.733 & 0.716 & -0.0249 & LN886600 \\
\hline & & R:GTCTGTGATACTGAGGTTCAAGTG & & & & & & & \\
\hline \multirow[t]{2}{*}{ BLS-205 } & $(\mathrm{CT})_{9}$ & F:ACTTCTCTTATTGCAAACCAGC & $156-180$ & FAM & 7 & 0.500 & 0.451 & -0.1111 & LN886601 \\
\hline & & R:AGTTCCATGACAAGGCCAAC & & & & & & & \\
\hline \multirow[t]{2}{*}{ BLS-208 } & $(\mathrm{CT})_{10}$ & F:GATGCTGACCACATCCCAG & $269-275$ & HEX & 4 & 0.360 & 0.522 & 0.3143 & LN886602 \\
\hline & & R:TGGACTTGATTTAGAGGTTTGCAG & & & & & & & \\
\hline \multirow[t]{2}{*}{ BLS-211 } & $(\mathrm{AG})_{10}$ & F:CTTGCCTGCTTGTGTGGAC & $165-171$ & FAM & 4 & 0.400 & 0.397 & -0.0087 & LN886603 \\
\hline & & R:AGCTGTCGATGGTTATGTTTC & & & & & & & \\
\hline \multirow[t]{2}{*}{ BLS-212 } & $(\mathrm{AC})_{10}$ & F:AGTGGACGTCAGTTGAGGG & $170-174$ & FAM & 3 & 0.731 & 0.639 & -0.1473 & LN886604 \\
\hline & & R:ACAGGAATGCAATTAGACGC & & & & & & & \\
\hline \multirow[t]{2}{*}{ BLS-213 } & (CT) 9 & F:GTGATTCAACGACAGTTACATTTCC & $200-206$ & HEX & 4 & 0.577 & 0.544 & -0.0608 & LN886605 \\
\hline & & R:TGAAAGGTTTGCTTCAGGTCG & & & & & & & \\
\hline \multirow[t]{2}{*}{ BLS-218 } & $(\mathrm{AT})_{8}$ & F:AATGCGAACCAAGACGGTG & $160-166$ & FAM & 4 & 0.233 & 0.271 & 0.1398 & LN886606 \\
\hline & & R:ATTGGCAACTAAACGGAGATG & & & & & & & \\
\hline \multirow[t]{2}{*}{ BLS-221 } & $(\mathrm{AG})_{10}$ & F:TGAGTTCCATGAAGAACGATGC & $185-191$ & FAM & 4 & 0.679 & 0.607 & -0.1201 & LN886607 \\
\hline & & R:AGATCTGGCCATACAAAGCG & & & & & & & \\
\hline \multirow[t]{2}{*}{ BLS-227 } & $(\mathrm{AG})_{11}$ & F:GGGCCACATGAGGGATTTG & $196-218$ & FAM & 9 & 0.857 & 0.833 & -0.0294 & LN886608 \\
\hline & & R:GCCCATCTTTCCTGGATGC & & & & & & & \\
\hline \multirow[t]{2}{*}{ BLS-236 } & $(\mathrm{CT}) 11$ & F:TCCCTAGCTCTACCGCAAC & $249-251$ & HEX & 2 & 0.567 & 0.494 & -0.1492 & LN886609 \\
\hline & & R:TGTACAATGCCAGCGCAAC & & & & & & & \\
\hline \multirow[t]{2}{*}{ BLS-238 } & $(\mathrm{AG})_{10}$ & F:TTTACCCACGCGCCTTAGC & $155-159$ & FAM & 3 & 0.310 & 0.376 & 0.1765 & LN886610 \\
\hline & & R:CGATGCCCGAACCCATTTG & & & & & & & \\
\hline \multirow[t]{2}{*}{ BLS-242 } & $(\mathrm{AG})_{10}$ & F:ACATGAAGCAAAGCAAATTACCC & $183-211$ & FAM & 10 & 0.655 & 0.804 & 0.1878 & LN886611 \\
\hline & & R:CCTTCTCTCCCTTTGAGCAAG & & & & & & & \\
\hline \multirow[t]{2}{*}{ BLS-243 } & $(\mathrm{AG})_{9}$ & F:CAGTTGGGTGTTGCAGGC & $149-163$ & FAM & 7 & 0.767 & 0.763 & -0.0053 & LN886612 \\
\hline & & $\mathrm{R}:$ GACGACTCAAACCTCAGTTAGC & & & & & & & \\
\hline \multirow[t]{2}{*}{ BLS-245 } & $(\mathrm{AT})_{8}$ & F:AGGTGATACTAACCATTGAGCG & $269-299$ & HEX & 9 & 0.852 & 0.811 & -0.0519 & LN886613 \\
\hline & & R:ACCATAATGTAGAACATCAATTGCC & & & & & & & \\
\hline \multirow[t]{2}{*}{ BLS-246 } & $(\mathrm{AG})_{10}$ & F:TGCACTCCCATTAAGCTGG & $171-205$ & FAM & 7 & 0.759 & 0.691 & -0.0990 & LN886614 \\
\hline & & R:CGGCTTCCTGCAACGAATG & & & & & & & \\
\hline
\end{tabular}

All loci use the same annealing temperature of $55^{\circ} \mathrm{C} . N_{\mathrm{A}}$ : number of alleles; $H_{\mathrm{O}}$ : observed heterozygosity; $H_{\mathrm{E}}$ : unbiased expected heterozygosity; $F$ : fixation index. *Indicates a deviation at $\mathrm{P}<0.05$ from Hardy-Weinberg equilibrium after Bonferroni correction.

Parameters of genetic diversity for 63 loci were calculated using GENEPOP v. 4.3 (Rousset, 2008). The same software was also used to detect deviations from Hardy-Weinberg equilibrium (HWE) and genotypic linkage disequilibrium (LD) among all pairs of loci. The significance levels of HWE and LD tests were adjusted using Holm's sequential Bonferroni correction (Holm, 1979).

\section{RESULTS AND DISCUSSION}

In total, 63 microsatellites showed polymorphisms. The number of alleles per locus varied from 2 to 16 (Table 1). The $H_{\mathrm{O}}$ and $H_{\mathrm{E}}$ ranged from 0.133 to 0.967 and from 0.127 to 0.912 , respectively. Three loci (BLS-76, BLS-134, and BLS-193) showed significant deviations from HWE, which was caused by heterozygote deficiency. After Bonferroni correction, significant LD was only detected in three locus pairs, loci BLS-53 and BLS-57, BLS-128 and BLS-243, and BLS-13 and BLS-212.

The identified polymorphic loci are useful to investigate genetic diversity and mating system of this species. These genetic markers may also be used to investigate the fine-scale spatial genetic structure and gene flow in NKMNR and HPNNR. All loci will provide valuable information for sound conservation of this species. In this study, the 63 microsatellites were tested individually. To reduce costs and save time, multiplex PCRs will be tested for these microsatellites for large samples in the future.

Genetics and Molecular Research 15 (3): gmr.15038234 


\section{Conflicts of interest}

The authors declare no conflict of interest.

\section{ACKNOWLEDGMENTS}

Research supported by the National Natural Science Foundation of China (\#31170196).

\section{REFERENCES}

Catchen JM, Amores A, Hohenlohe P, Crecko W, et al. (2011). Stacks: building and genotyping loci de novo from shortread sequences. G3 (Bethesda) 1: 171-182. http://dx.doi.org/10.1534/g3.111.000240

Catchen J, Hohenlohe PA, Bassham S, Amores A, et al. (2013). Stacks: an analysis tool set for population genomics. Mol. Ecol. 22: 3124-3140. http://dx.doi.org/10.1111/mec.12354

Doyle JJ (1991). DNA protocols for plants-CTAB total DNA isolation. In: Molecular techniques in taxonomy (Hewitt GM and Johnston A, eds.). Springer-Verlag, Berlin, 283-293.

Faircloth BC (2008). MSATCOMMANDER: detection of microsatellite repeat arrays and automated, locus-specific primer design. Mol. Ecol. Resour. 8: 92-94. http://dx.doi.org/10.1111/j.1471-8286.2007.01884.x

Fu L and Jin J (1992). China plant red data book-rare and endangered plants. Vol. 1. Science Press, Beijing.

Holm S (1979). A simple sequentially rejective multiple test procedure. Scand. J. Stat. 6: 65-70.

Liang Y, Xu G, Zhang H, Wu X, et al. (2013). Genetic diversity of natural and planted populations of Bretschneidera sinensis from Nanling region. Sci. Silvae Sin. 48: 45-52.

Qiao Q, Xing F, Chen H and Wang M. (2011). Research progress and direction in Bretschneidara sinensis, a rare endemic plant in China. Chin. Wild Plant Resour. 30: 4-8.

Qiao Q, Chen H, Xing F, Wang F, et al. (2012). Pollination ecology of Bretschneidera sinensis (Hemsley), a rare and endangered tree in China. Pak. J. Bot. 44: 1897-1903.

Rousset F (2008). GENEPOP'007: a complete re-implementation of the GENEPOP software for Windows and Linux. Mol. Ecol. Resour. 8: 103-106. http://dx.doi.org/10.1111/j.1471-8286.2007.01931.x

Sun W (1998): Bretschneidera sinensis. The IUCN red list of threatened species 1998: T32324A9697750.

Xu G, Liang Y, Jiang Y, Liu X, et al. (2013). Genetic diversity and population structure of Bretschneidera sinensis, an endangered species. Biodivers. Sci. 21: 723-731.

Genetics and Molecular Research 15 (3): gmr.15038234 